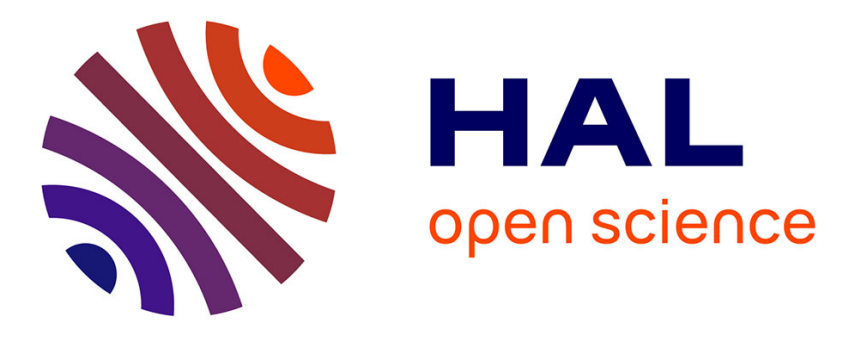

\title{
Micromagnetic Modeling of Magneto-Mechanical Behavior
}

Frédérick Sorel Mballa-Mballa, Olivier Hubert, Song He, Sophie Depeyre, Philip Meilland

\section{- To cite this version:}

Frédérick Sorel Mballa-Mballa, Olivier Hubert, Song He, Sophie Depeyre, Philip Meilland. Micromagnetic Modeling of Magneto-Mechanical Behavior. IEEE Transactions on Magnetics, 2014, 50, pp.1 4. 10.1109/TMAG.2013.2288911 . hal-01532888

\section{HAL Id: hal-01532888 \\ https://hal.science/hal-01532888}

Submitted on 4 Jun 2017

HAL is a multi-disciplinary open access archive for the deposit and dissemination of scientific research documents, whether they are published or not. The documents may come from teaching and research institutions in France or abroad, or from public or private research centers.
L'archive ouverte pluridisciplinaire HAL, est destinée au dépôt et à la diffusion de documents scientifiques de niveau recherche, publiés ou non, émanant des établissements d'enseignement et de recherche français ou étrangers, des laboratoires publics ou privés. 


\title{
Micromagnetic modeling of magneto-mechanical behavior
}

\author{
Frédérick Sorel Mballa-Mballa*†, Olivier Hubert* ${ }^{*}$, Song He ${ }^{\ddagger}$, Sophie Depeyre ${ }^{\ddagger}$ and Philip Meilland ${ }^{\dagger}$ \\ *LMT-Cachan (ENS Cachan, CNRS (UMR8535), UPMC, Univ Paris-Sud) \\ 61 avenue du Président Wilson 94235 Cachan, France \\ $\dagger$ ARCELORMITTAL, AMMR Measurement \& Control \\ Voie Romaine BP 30320 F-57283 Maizières-lès-Metz Cedex, France \\ * Pôle Universitaire Léonard de Vinci \\ 92916 Paris La Défense Cedex, France \\ Email: mballa@1mt.ens-cachan.fr, hubert@1mt.ens-cachan.fr,philip.meilland@arcelormittal.com, \\ song.he@devinci.fr, sophie.depeyre@devinci.fr
}

\begin{abstract}
A magneto-mechanical static modeling of ferromagnetic particle based on minimization of an energy function is presented. This modeling is made of a conjugate gradient method coupled with finite element method for the mechanical problem resolution. 2D computational results highlighting the influence of magneto-mechanical coupling on the magnetic microstructure and behavior are reported.
\end{abstract}

\section{INTRODUCTION}

The micromagnetic theory [1] is usually used to describe the non-linear behavior of ferromagnetic media. If micromagnetic modeling of magnetization process and domains structure are now well mastered, the description of magnetostriction and associated magneto-mechanic interactions remains unachieved or badly implemented in the literature [2]. Indeed modeling the intrinsic stress due to a free strain like magnetostriction is rather complex. A mechanical approach of this problem is relevant. An uncoupled micromagnetic modeling is presented in a first part. The modeling of magneto elastic coupling is achieved thanks to the introduction of elastic and magnetoelastic interactions depending on stress and magnetization in the free energy expression.

\section{Magnetic BehaVior AND ENERGEtic BALANCE}

The mechanism of magnetization originates from the existence of intragranular microstructure organized in domains associated to magnetization $\vec{m}=M_{s} \alpha_{i} \cdot \vec{e}_{i}$ (with $\alpha_{i}$ the direction cosines of the magnetization and $M_{s}$ the saturation magnetization) and separated by walls. At the microscopic scale, the division into domains results from energy balance between different elementary contributions. Three contributions in particular are at the origin of this subdivision: the exchange energy, the magnetocrystalline energy and dipole interaction [5]. The exchange energy in a medium $\Omega$ usually involves a potential of the following form with exchange constant $A$ :

$$
\mathscr{E}_{e x}=\int_{\Omega} A \cdot\|\overline{\bar{\nabla}} \vec{m}\|^{2} d \Omega
$$

The magneto-crystalline energy is due to an interaction of the magnetic moments with crystal axes $\vec{d}$. This interaction takes the form of a function $\Psi(\vec{d}, \vec{m})$. For a cubic symmetry, magnetocrystalline energy is given by:

$$
\mathscr{E}_{a}=\int_{\Omega} K_{0}+K_{1}\left(\alpha_{1}^{2} \alpha_{2}^{2}+\alpha_{2}^{2} \alpha_{3}^{2}+\alpha_{1}^{2} \alpha_{3}^{2}\right)+K_{2}\left(\alpha_{1}^{2} \alpha_{2}^{2} \alpha_{3}^{2}\right) d \Omega
$$

with $K_{i}$ the magneto crystalline anisotropy constants.

The dipolar interaction plays a fundamental role in the formation of domains. It is related to the mutual interaction of the magnetic moments at position $\vec{r}$ with each others and to the geometry of $\Omega$. This field called demagnetizing field $\vec{H}_{d}(\vec{m})$ satisfies the Maxwell equations $\left(\vec{\nabla} \wedge \vec{H}_{d}(\vec{m})=\overrightarrow{0}\right)$ and then derives from a scalar potential $\zeta$ satisfying the following equations:

$$
\begin{gathered}
\vec{H}_{d}(\vec{m})=-\vec{\nabla} \zeta \\
\Delta \zeta(\vec{r})=\frac{1}{\mu_{0}} \nabla \vec{m}(\vec{r}) \forall \vec{r} \in \Omega \\
\Delta \zeta(\vec{r})=0 \forall \vec{r} \in \mathbb{R}^{3}-\Omega \\
{[\zeta]=0 \forall \vec{r} \in \partial \Omega}
\end{gathered}
$$

$\mu_{0}$ is the vacuum permeability and $\zeta$ is the solution of a Poisson problem (4) and reflects the non local form of the demagnetizing field. The calculation of this quantity is usually very time-consuming. The demagnetizing energy $\mathscr{E}_{d}$ is usually associated to a potential of the form:

$$
\mathscr{E}_{d}=\int_{\Omega}-\frac{\mu_{0}}{2} \vec{H}_{d}(\vec{m}) \cdot \vec{m} d \Omega
$$

The last interaction is the Zeeman energy that reflects the influence of the applied field $\vec{H}_{\text {ext }}$ on the magnetic moments:

$$
\mathscr{E}_{h}=-\int_{\Omega} \mu_{0} \vec{H}_{e x t} \cdot \vec{m} d \Omega
$$




\section{Micromagnetic Modeling}

Micromagnetism is a theoretical approach to describe the process of magnetization at a scale large enough to replace the atomic magnetic moments by continuous functions, and small enough to account for the transition zones between magnetic domains [1][6]. Approximation is obtained thanks to the minimization of an energy functional. This minimization with respect to the magnetization gives rise to a state of metastable equilibrium associated with a particular configuration of the magnetic structure. The contribution of Brown[1] was to define an expression of the free energy $\mathscr{E}_{t o t}$ as the sum over a volume $\Omega$ of internal and external contributions mentioned above.

$$
\mathscr{E}_{t o t}(\vec{m})=\mathscr{E}_{h}+\mathscr{E}_{e x}+\mathscr{E}_{a}+\mathscr{E}_{d}
$$

At equilibrium, the relation of energy stationarity must be satisfied:

$$
\vec{m}(\vec{r})=\operatorname{Min}_{\left(\vec{m} \in \mathbb{R}^{n}\right)}\left(\mathscr{E}_{t o t}(\vec{m})\right) \quad \forall \vec{r} \in \Omega
$$

under the constraint:

$$
\|\vec{m}\|=M_{s} \forall \vec{r} \in \Omega
$$

For a small variation of the local magnetization $\delta \vec{m}$, the variation of the total free energy is given by:

$$
\delta \mathscr{E}_{t o t}(\vec{m})=\mathscr{E}_{t o t}(\vec{m}+\delta \vec{m})-\mathscr{E}_{t o t}(\vec{m})=0
$$

so that:

$$
\begin{gathered}
\delta \mathscr{E}_{t o t}(\vec{m}) \approx \int_{\Omega}\left(-\mu_{0} \vec{H}_{e x t}-\mu_{0} \vec{H}_{d}+\frac{\partial \Psi(\vec{d} \cdot \vec{m})}{\partial \vec{m}}\right. \\
\left.+\mu_{0} A \Delta \vec{m}\right) \cdot \delta \vec{m} d \Omega+\oint \mu_{0} A \frac{\partial \vec{m}}{\partial \vec{n}} \cdot \delta \vec{m} \quad \partial \Omega
\end{gathered}
$$

where $\vec{n}$ is the unit vector normal to the surface $\partial \Omega$ of the volume $\Omega$. The effective field $\vec{H}_{e f f}$ defined by equation (12) is the local magnetic field experienced by each magnetic moment.

$$
\vec{H}_{e f f}=\vec{H}_{e x t}+\vec{H}_{d}-A \Delta \vec{m}-\frac{1}{2} \frac{\partial \Psi(\vec{d} . \vec{m})}{\partial \vec{m}}
$$

The condition of stationarity of the total energy can thus be written in the following form:

$$
\delta \mathscr{E}_{t o t}(\vec{m})=-\int_{\Omega} \mu_{0} \vec{H}_{e f f} . \delta \vec{m} d \Omega+\oint \mu_{0} A \frac{\partial \vec{m}}{\partial \vec{n}} \cdot \delta \vec{m} \quad \partial \Omega=0
$$

From (13), a system called "Brown System" [1] is obtained that defines the two following conditions to be met at equilibrium:

$$
\begin{aligned}
& \vec{m} \wedge \vec{H}_{e f f}=\overrightarrow{0} \quad \forall \quad \vec{x} \in \Omega \\
& \vec{m} \wedge \frac{\partial \vec{m}}{\partial \vec{n}}=\overrightarrow{0} \quad \forall \quad \vec{x} \in \partial \Omega
\end{aligned}
$$

The estimation of the total free energy is made thanks to a finite volume discretization of equation (7). An iterative conjugate gradient method is used to solve the optimization problem (8). More details are available in [3] and [4].

\section{INTRODUCTION OF MAGNETO-ELASTIC COUPLING}

The change of magnetization of a magnetic medium induces a deformation $\varepsilon^{\mu}$ called magnetostriction. It is a spontaneous deformation that only depends on the magnetic state of the material. Magnetization direction $\vec{m}$ and magnetostriction are linked by a non-linear relationship. The magnetostriction strain tensor for cubic symmetry is isochorus and given by:

$$
\varepsilon^{\mu}=\frac{3}{2}\left(\begin{array}{ccc}
\lambda_{100}\left(\alpha_{1}^{2}-\frac{1}{3}\right) & \lambda_{111} \alpha_{1} \gamma_{2} & \lambda_{11 \alpha 1} \alpha_{1} \alpha_{3} \\
\lambda_{111} \alpha_{1} \alpha_{2} & \lambda_{100}\left(\alpha_{2}^{2}-\frac{1}{3}\right) & \lambda_{111} \alpha_{2} \alpha_{3} \\
\lambda_{111} \alpha_{1} \alpha_{3} & \lambda_{111} \alpha_{2} \alpha_{3} & \lambda_{100}\left(\alpha_{3}^{2}-\frac{1}{3}\right)
\end{array}\right)
$$

where $\lambda_{100}$ and $\lambda_{111}$ are two magnetostrictive constants (deformation along $<100\rangle$ and $<111>$ crystallographic axes). This deformation is usually incompatible (i.e. not deriving from a displacement field). The elastic deformation $\varepsilon^{e}$ of the magnetic medium must then correct this incompatibility. It results to a stress field even in absence of any external mechanical loading. When the magnetostriction $\varepsilon^{\mu}$ is known for a medium, the total deformation $\varepsilon$ is obtained by simple addition $\left(\varepsilon=\varepsilon^{\mu}+\varepsilon^{e}\right)$ since small pertubation assumption can be applied. The total deformation $\varepsilon$ derives from a displacement field $\vec{u}(16)$ and stress field $\sigma$ associated to elastic deformation obeys to the equilibrium equation (17) with $\mathbb{C}$ the stiffness tensor of the medium.

$$
\begin{gathered}
\varepsilon=\frac{1}{2}\left(\overline{\bar{\nabla}} \vec{u}+{ }^{t} \overline{\bar{\nabla}} \vec{u}\right) \quad \text { in } \Omega \\
\vec{\nabla} \cdot \sigma=\overrightarrow{0} \text { in } \Omega \\
\sigma=\mathbb{C}: \varepsilon^{e} \text { in } \Omega
\end{gathered}
$$

The boundary conditions of the problem are given by:

$$
\begin{gathered}
\sigma . \vec{n}=\vec{T}_{d} \quad \text { on } \quad \partial \Omega_{t} \\
\vec{u}=\vec{u}_{d} \quad \text { on } \quad \partial \Omega_{u}
\end{gathered}
$$

$\vec{T}_{d}$ and $\vec{u}_{d}$ are the surface forces and displacements applied at the boundaries $\partial \Omega_{t}$ and $\partial \Omega_{u}$ respectively. The additivity of deformations allows to reformulate the mechanical balance to introduce an internal stress of magnetostrictive origin:

$$
\vec{\nabla} \cdot \sigma^{\star}-\vec{f}^{\mu}=\overrightarrow{0}
$$

$\sigma^{\star}$ is the total stress and $\vec{f}^{\mu}=\vec{\nabla}\left(\mathbb{C}: \varepsilon^{\mu}\right)$ the force density of magnetostrictive origin. The mechanical problem can also be reduced to an optimization problem where the displacement field minimizes the elastic energy $\mathscr{E}_{\sigma}$ given by the application of a variational formulation of the problem (19)

$$
\vec{u}=\operatorname{Min}_{\left(\vec{v} \in \mathscr{H}_{\Omega}^{1}\right)} \mathscr{E}_{\sigma}(\vec{v}) \quad \text { with } \vec{v}=\vec{u}_{d} \quad \text { on } \quad \partial \Omega_{d}
$$

with:

$$
\mathscr{E}_{\sigma}(\vec{v})=\frac{1}{2} \varepsilon(\vec{v}): \mathbb{C}: \varepsilon(\vec{v})-\varepsilon(\vec{v}): \mathbb{C}: \varepsilon^{\mu}
$$

In the case of Finite Element Modeling (FEM) with imposed displacement, the minimization problem (20) can be simplified 
in the form of a linear system (22) where $\mathbf{K}, \hat{U}$ and $\hat{F}$ are the stiffness matrix of the system, the generalized displacement vector and the generalized force vector corresponding to forces of magnetostrictive origin respectively.

$$
\mathbf{K} . \hat{U}=\hat{F}
$$

Equation 23 gives a discretized formulation the terms of equation 22 for linear form functions $\phi$ and isoparametric finite elements on domain $\Omega_{h}$.

$$
\begin{gathered}
\mathbf{K}_{h}=\sum_{i=1}^{N_{h}} \sum_{j=1}^{N_{h}} \int_{\Omega_{h}} \vec{\nabla} \phi_{j}: \mathbb{C}: \vec{\nabla} \phi_{i} d \Omega_{h} \\
\hat{F}_{h}=\sum_{j=1}^{N_{h}} \int_{\Omega_{h}} \varepsilon^{\mu}: \mathbb{C}: \vec{\nabla} \phi_{j} d \Omega_{h} \\
\hat{U}_{h}=\sum_{j=1}^{N_{h}} u_{j}
\end{gathered}
$$

Considering a magneto-mechanical coupled approach, contributions related to magnetic equilibrium, but also contributions related to the mechanical equilibrium and various interactions between these two phenomena are involved in the energy equilibrium. The free energy that suitably describes a deformable magnetic medium takes the following form:

$$
\mathscr{E}_{t o t}(\vec{m}, \vec{u})=\mathscr{E}_{h}+\mathscr{E}_{e x}+\mathscr{E}_{a}+\mathscr{E}_{d}+\mathscr{E}_{\sigma}
$$

The stability condition of the energy is obtained if and only if the magnetization and displacement fields minimize the total free energy simultaneously.

A condition of minimization is the cancellation of all the partial derivatives independently. We solve the system:

$$
\frac{\partial \mathscr{E}_{\text {tot }}}{\partial \vec{m}}=\overrightarrow{0} \quad \frac{\partial \mathscr{E}_{\text {tot }}}{\partial \vec{u}}=\overrightarrow{0} \quad \forall \vec{x} \in \Omega
$$

The first minimization always provides the torque condition given by equation (14). The second provides the linear system corresponding to equation (22). In addition the effective field is complemented by a term called elastic induced field, and linked to the derivation of the magneto-elastic energy compared to magnetization:

$\vec{H}_{e f f}=\vec{H}_{e x t}+\vec{H}_{d}-A \Delta \vec{m}-\frac{1}{2} \frac{\partial \Psi(\vec{d} \cdot \vec{m})}{\partial \vec{m}}-\vec{H}_{\sigma}=-\frac{1}{\mu_{0}} \frac{\partial \mathscr{E}_{t o t}(\vec{m})}{\partial \vec{m}}$

with:

$$
\vec{H}_{\sigma}=-\frac{1}{\mu_{0}} \frac{\partial \mathscr{E}_{\sigma}}{\partial \vec{m}}=-\frac{1}{\mu_{0}} \frac{\partial\left(-\sigma: \varepsilon^{\mu}\right)}{\partial \vec{m}}
$$

Using the magnetostriction definition (15), the elastic induced field becomes:

$$
\vec{H}_{\sigma_{i}}=\frac{3}{\mu_{0}}\left(\lambda_{100} \sigma_{i i} \alpha_{i}+\lambda_{111} \sum_{j \neq i} \sigma_{i j} \alpha_{j}\right)
$$

The calculation of contribution to the effective field at each iteration step requires to know the stress field solving the equilibrium equations (17) and (18). The solution is obtained by an inversion of the linear system (22). We use algorithm 1 that is the so-called "constrained" approach.

Assuming: $\vec{m}_{0}, \delta \mathscr{E}_{\text {tot }}\left(\vec{m}_{0}\right)=\vec{g}_{0}, \vec{w}_{0}=\vec{g}_{0}, \mathbb{K}_{h} \cdot U_{h_{0}}=F_{h_{0}}$;

while $n \geq 1$, do

Find $\rho_{n} \in \mathbb{R}$ so that $\forall \rho \in \mathbb{R}$;

$\mathscr{E}_{\text {tot }}\left(\frac{\vec{m}_{n}-\rho_{n} \vec{w}_{n}}{\left\|\vec{m}_{n}-\rho_{n} \vec{w}_{n}\right\|}, \sigma_{n}\right)<\mathscr{E}_{\text {tot }}\left(\frac{\vec{m}_{n}-\rho \vec{w}_{n}}{\left\|\vec{m}_{n}-\rho \vec{w}_{n}\right\|}, \sigma_{n}\right)$;

$\vec{m}_{n+1}=\frac{\vec{m}_{n}-\rho_{n} \vec{w}_{n}}{\left\|\vec{m}_{n}-\rho_{n} \vec{w}_{n}\right\|}$

$\mathbb{K}_{h} \cdot U_{h_{n+1}}=F_{h_{n+1}}$;

$\vec{g}_{n+1}=\vec{H}_{e f f}\left(\vec{m}_{n+1}, \sigma_{n+1}\right)$;

$\vec{w}_{n+1}=\vec{g}_{n+1}+r_{n} \vec{w}_{n}$

Where $r_{n}=\frac{\vec{g}_{n+1} \cdot\left(\vec{g}_{n+1}-\vec{g}_{n}\right)}{\left\|\vec{g}_{n}\right\|_{\mathbb{P}^{n}}^{2}}$;

end

Algorithm 1: Modified conjugate gradient for "constraint" magneto-mechanical problem.

\section{ApPLICATIONS}

Micromagnetic code "sivimm $2 d$ " [3] is used as the basis for the implementation of the magneto-mechanical formulation. We study the evolution of domains structure of a ferromagnetic crystal with cubic symmetry and associated magnetomechanical behavior (axes: $[100]=\vec{x}$ and $[010]=\vec{y}$ ). The region of interest is a square of $30 \times 30 \mu \mathrm{m}^{2}$ in the vacuum. This region is meshed by 3996 triangular elements. Magnetic and mechanical constants are reported on table $\mathrm{I}^{1}$.

\begin{tabular}{|c|c|c|}
\hline$M_{s}=1.71 \times 10^{6} \mathrm{~A} . \mathrm{m}^{-1}$ & $A=1.8 \times 10^{-11} \mathrm{~J} . \mathrm{m}^{-1}$ & $K_{1}=48 \times 10^{3} \mathrm{~J} . \mathrm{m}^{-3}$ \\
$K_{2}=0 \mathrm{~J} . \mathrm{m}^{-3}$ & $\lambda_{100}=21 \times 10^{-6}$ & $\lambda_{111}=-21 \times 10^{-6}$ \\
$C_{11}=228 \mathrm{GPa}$ & $C_{12}=132 \mathrm{GPa}$ & $C_{44}=116.5 \mathrm{GPa}$ \\
\hline
\end{tabular}

TABLE I

PHYSICAL CONSTANTS USED FOR SIMULATIONS

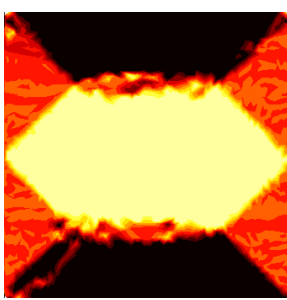

(a) Uncoupled

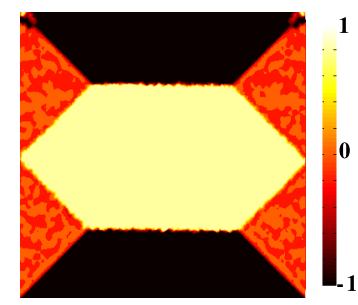

(b) With ME coupling
Fig. 1. Influence of magneto-elastic coupling on initial domain definition $\left(H_{\text {ext }}=0\right)$ : color code corresponding to $m_{x}=(\vec{m} \cdot \vec{x})$

The first simulations presented allows to highlight the contribution of the magneto-mechanical coupling to the domain definition (Fig. 1): Indeed, in the absence of magneto-elastic effect, the magnetic microstructure is dominated by demagnetizing effects and magnetocrystalline anisotropy. Stress acts as an additional source of anisotropy that stabilizes the magnetic microstructure $^{2}$. Figure 2 illustrates the change of domain

${ }^{1}$ Due to $2 \mathrm{D}$ restriction, the plane stress assumption $\left(\sigma_{z z}=0\right)$ is used, that requires the redefinition of the stiffness tensor $\mathbb{C}$

${ }^{2}$ The elastic energy density is reaching $200 \mathrm{~J} \cdot \mathrm{m}^{-3}$ in the walls. The thickness decrease of $180^{\circ}$ walls leads to a minimizing of this energy term. 


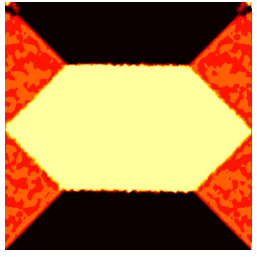

(a) $H_{\text {ext }}=0.0$

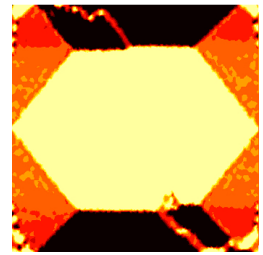

(b) $H_{\text {ext }}=0.15 M_{s}$

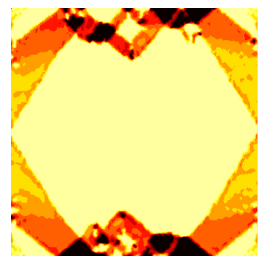

(c) $H_{\text {ext }}=0.3 M_{s}$

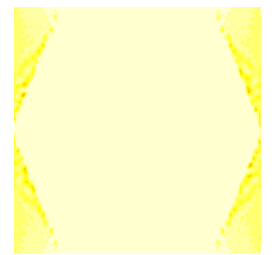

(d) $H_{\text {ext }}=0.48 M_{s}$ (e) $H_{\text {ext }}=0.9 M_{S}$

Fig. 2. Change of domain structure with increasing magnetic field $(\vec{m} \cdot \vec{x})$

structure with increasing magnetic field along $\vec{x}$ axis and figure 3 shows the associated magnetic and magnetostrictive behavior (long. magnetostriction $E_{x x}^{\mu}$ and trans. magnetostriction $E_{y y}^{\mu}$ ). This figure exhibits the large demagnetizing effect on the 2D square structure and effect of initial domains configuration on magnetostriction amplitude.

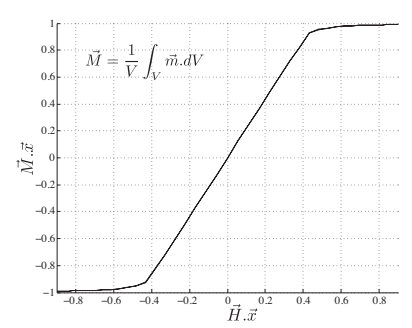

(a) Magnetization $\vec{M} \cdot \vec{x}$

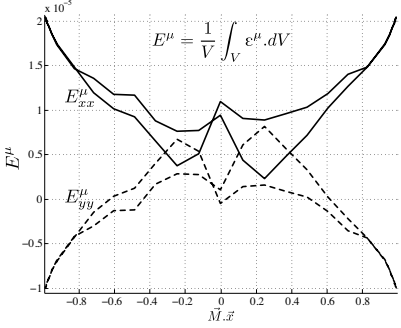

(b) Longitudinal $E_{x x}^{\mu}$ and transversal $E_{y y}^{\mu}$ magnetostriction
Fig. 3. Average magnetic and magnetostrictive behavior of iron single crystal over the volume $V$.

The magneto mechanical coupling introduced above allows the opportunity to explore the influence of external mechanical loading on the domain configuration and behavior. The figure 4 illustrates the effect of applied displacement in the direction $\vec{x}$ on the magnetic microstructure at zero magnetic field. $\Sigma_{x x}$ defines the average applied stress corresponding to the imposed displacement. It exhibits the so-called refinement of domain structure with stress [5]. Note that the stress required for changing the domain patterns is very high due to demagnetizing effects ${ }^{3}$ and that re-meshing has not been performed $^{4}$. As a final illustration, figure 5 shows a simulation of the so-called $\Delta E$ effect, i.e. change of magnetostriction with stress at zero external magnetic field.

\footnotetext{
${ }^{3}$ The demagnetizing factor is estimated to 0.46 . It leads to large magnetic field and so large stress magnitudes to move the domain microstructure. 10GPa is obviously unrealistic comparing to standard yield stress of iron.

${ }^{4}$ given the stress levels, the displacements are large enough to require the re-meshing. This must clearly be improved.
}

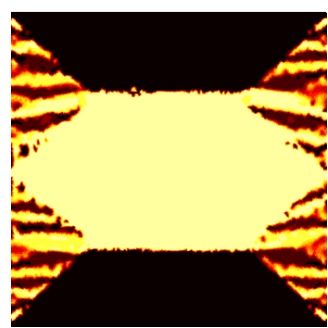

(a) $m_{x}=\vec{m} \cdot \vec{x}$

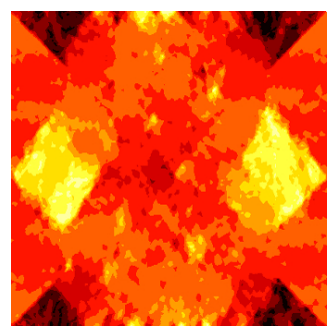

(c) $m_{x}=\vec{m} \cdot \vec{x}$

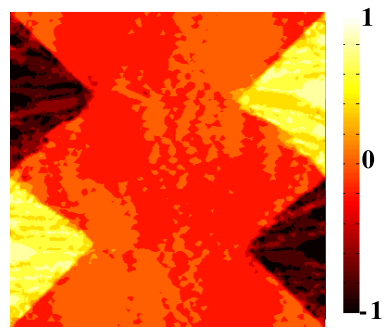

(b) $m_{y}=\vec{m} \cdot \vec{y}$

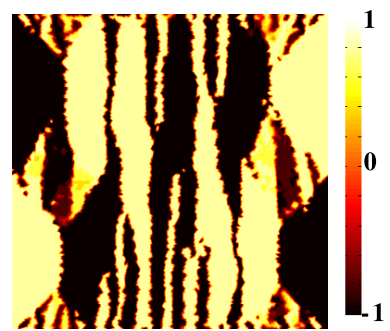

(d) $m_{y}=\vec{m} \cdot \vec{y}$
Fig. 4. Effect of applied stress along direction $\vec{x}$ on domain patterns: (a,b) average tensile stress: $\Sigma_{x x}=9.62 G P a \quad \Sigma_{y y}=\Sigma_{x y}=0$; (c,d) average compressive stress $\Sigma_{x x}=-9.62 \mathrm{GPa} \quad \Sigma_{y y}=\Sigma_{x y}=0$ at zero applied field.

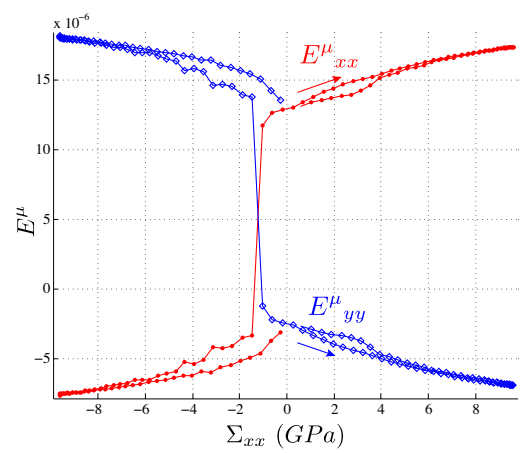

Fig. 5. Illustration of $\Delta E$ effect: average longitudinal $E_{x x}^{\mu}$ and transversal $E_{y y}^{\mu}$ magnetostriction as function of average axial stress $\Sigma_{x x}$ for zero applied field

\section{CONCLUSION}

The implementation of the full magneto-elastic coupling in a micromagnetic calculation is effectiveness. This introduction has the property to stabilize the magnetic microstructure and allows the prediction of magnetostriction, $\Delta E$ effect and change of magnetic susceptibility with stress. Simulation of $2 \mathrm{D}$ polycrystals and $3 \mathrm{D}$ single crystals is effectiveness too but calculation requires very high computation time so that only small problems can be solved at present.

\section{REFERENCES}

[1] W. F. Brown. Micromagnetics, John Wiley \& Sons, New York, 1963.

[2] Y. C. Shu, M. P. Lin, K. C. Wu., Mechanics of Materials (36), pp. 975997, 2004.

[3] M. Bernadou, S. Depeyre, S. He and P. Meilland, J. of intel. material syst.and struct., 16, pp. 1015-1023, 2005.

[4] F. Mballa Mballa, PhD thesis, ENS-Cachan, 2013.

[5] A. Hubert, R. Schäfer. Magnetic Domains. The analysis of magnetic microstructures, Springer, 2008.

[6] A. Aharoni Introduction to the Theory of Ferromagnetism. Oxford University Press, New York, 1996. 